\title{
¿Quién hace al patrimonio? Su valoración y uso desde la perspectiva del campo de poder
}

\author{
Mauricio Benjamín Jiménez Ramírez \\ Mariana Sainz Navarro
}

Introducción

- I restaurador, al intervenir una pieza, no sólo se adentra en los misterios
de las transformaciones de los materiales que la componen, sino también
participa de las que experimentan su significado y valoración, lo que le permite interpretar los motivos por los que se conserva y se da a conocer como objeto paradigmático de una cultura. ${ }^{1}$ Sin embargo, con mucha frecuencia, esta evaluación sobre los valores y significados de un bien cultural es llevada a cabo con poco interés y llega a la conclusión de que los valores de la obra son únicos, inmutables y sin más detalles específicos. Esto es en parte efecto de una tradición teórica que da sustento a la restauración desde mediados del siglo xx y cuyo interés se centra en una autenticidad trascendental que la obra posee de forma independiente a las valoraciones que podamos hacer de ella (véase Brandi 1996).

La renovación teórica del campo de la conservación-restauración, aún incipiente, no ha terminado de evidenciar que las labores de restauración están definidas por una forma de ver los objetos culturales que, además de no corresponder necesariamente con la valoración que éstos tenían al ser creados, por supuesto altera, para bien o para mal, los valores que se le asignaban antes de la restauración. En toda intervención de restauración subyace una interpretación sobre cómo se debe observar y qué significa el objeto intervenido, la cual no es, en absoluto, producto de la mente independiente del restaurador, sino una construcción social con la que los especialistas comunican al resto de la sociedad, a partir de patrones de interpretación que han incorporado en su praxis, una forma canonizada de apreciar los bienes culturales. Las acciones de conservación y consumo de los bienes culturales constituyen así un campo donde interactúan diferentes actores, con reglas que todos deben respetar, si quieren disfrutar de los bienes culturales que están a cargo de los especialistas.

Se puede decir que la conservación de bienes culturales se ha constituido en un área o esfera relativamente autónoma donde se controlan ciertos objetos culturales -los cuales generalmente son bienes simbólicos producidos en otras épocas- y se han definido reglas que determinan el papel de aquellos que

1 García Canclini (1979:17) menciona que "el objeto de estudio de la estética y la historia no puede ser la obra, sino el proceso de circulación social en el que sus significados se constituyen y varían". 
quieren participar $\mathrm{o}$, al menos, tener un acercamiento a los bienes bajo su control. Esta situación es análoga a la que observó Pierre Bourdieu al formular su teoría de los campos.

En este artículo se pretende hacer un análisis de los problemas subyacentes en la conservación de un objeto, relacionados con la valoración que se hace de éste al momento de decidir su intervención. Este análisis, que parte de la perspectiva de que esta valoración es producto de las condiciones sociales objetivas en las que se desarroIlan las actividades de conservación, se propone, para ello, discutir el concepto de campo, desarrollado a partir de la teoría sociológica de Bourdieu, usando como objeto de análisis los efectos de las prácticas relacionadas con la restauración de bienes culturales en México, en particular aquellas encaminadas a la valoración o puesta en valor de las obras que se han de restaurar. ${ }^{2}$

En sentido estricto, hay que reconocer a la obra de $\mathrm{Al}$ fredo Vega Cárdenas (2008) haber introducido el trabajo de Bourdieu en el análisis de la restauración como práctica social, aunque, como aquel mismo aclara, su análisis se ha centrado en entender la dinámica de la disciplina: la Restauración como campo disciplinar (y, por lo mismo, con mayúsculas). Así, el modelo propuesto por este autor repara poco en las consecuencias de la configuración particular del campo en el ejercicio de la restauración.

\footnotetext{
${ }^{2}$ Consideramos el concepto de puesta en valor como está expuesto en algunos documentos internacionales sobre la materia. En particular, rescatamos el concepto de las Normas de Quito que, en resumen, identifican la puesta en valor con las acciones tendentes a la habilitación de un bien para asignarle nuevos usos a partir de la identificación de las características que permitan su aprovechamiento. Esta habilitación incluye la transferencia de conocimientos sobre el bien de los especialistas al resto de la sociedad para coadyuvar a su disfrute (1967). Como veremos más adelante, uno de los puntos centrales de nuestra discusión radica en esta transferencia de conocimientos. En documentos más recientes, aparece además el concepto de significación cultural (véase Carta de ıсомos Australia 1999), el cual se asume como sinónimo de valor de patrimonio cultural y que resume los valores asignados a un objeto por las "generaciones pasada, presente y futura", los que se pretenden mantener mediante la conservación. Sin embargo, para que ésta se dé, es necesaria una previa identificación de los valores. En resumen, podemos decir que, al menos en el campo de la conservación, la valoración se identifica con la determinación, hecha por los especialistas, de los valores, inmanentes o asignados, del bien cultural que se ha de conservar. No pasamos por alto que desde hace varios años hay un acalorado debate sobre la forma en que debería realizarse dicho proceso, especialmente a partir del reconocimiento de que la valoración debería de partir de la perspectiva de los creadores y custodios del patrimonio, como se expresa en el Documento de Nara (1994). En este sentido, se ha suscitado toda una corriente de intentos y metodologías más o menos logrados -como la animación sociocultural, la educación patrimonial, la museología participativa o el modelo de conservación, identidad y desarrollo- con la intención de democratizar o hacer participativo el proceso de valoración y consumo del patrimonio (Ariel Olmos 2004; Teixeira 2006; Tagliorette et al. 2008; Pérez Ruiz 2008; Arrieta Urtizberea 2008; Fontal Merillas 2003; Tenopala García 1998).
}

Aún más importante: el modelo de Vega Cárdenas del campo de la restauración está subsumido en el campo general del poder, pero no se relaciona claramente con el de la producción cultural, que es el que nos interesa particularmente. En su formulación original, la teoría del campo de poder es parte de un esfuerzo por comprender el campo de producción cultural y se complementa con la noción previa del habitus, ambas -campo de poder y habitus-, necesarias para entender el problema al que nos referimos.

Acerca del campo tenemos que decir de manera preliminar, para introducir al lector ajeno a la obra de Bourdieu, ${ }^{3}$ que se trata de un espacio relacional, esto es, aquel en el que diferentes actores ocupan posiciones que dependen de su relación con las posiciones relativas de los otros actores. Están interesados en ingresar en este espacio y ocupar determinadas posiciones porque en él se disputa el control de algo, a lo que genéricamente se le conoce como capital. La disputa por el capital no es arbitraria, sino que obedece a una serie de reglas definidas por los propios participantes, las cuales aprenden durante su participación en el campo hasta que las hacen parte de su propia personalidad, formando un habitus. Éste puede entenderse como el conjunto de reglas que un actor hace suyas para participar en el campo, pero que percibe no como reglas, sino como la condición de posibilidad para la existencia del campo, así como del sentido común que le da valor. ${ }^{4}$ $\mathrm{El}$ anterior, pese a ser un grueso resumen de las muchas sutilezas de este par de nociones, nos permite bosquejar cómo nos pueden ayudar a explicar los fenómenos que se dan en torno de la conservación del patrimonio.

\footnotetext{
3 Pierre Bourdieu nació en 1930 en Denguin, en los Pirineos Atlánticos, Francia. Comenzó su labor sociológica haciendo etnografía en Argelia, donde sentó las bases de sus trabajos posteriores. Su obra más celebre, La distinción (1998), es un estudio sobre la forma en que la posición social condiciona los juicios de gusto, desde la cultura, pero con obvias implicaciones políticas y económicas. Fue un duro crítico del sistema educativo y académico francés $y$, durante mucho tiempo, uno de los intelectuales más influyentes en su país y Europa. Entre sus conceptos centrales hay que señalar, por supuesto, el de campo, que ahora analizamos, así como el habitus, el capital cultural y social, la violencia simbólica, que fueron herramientas fundamentales para desvelar el papel de la cultura en la reproducción social. Murió en París a la edad de 71 años.

${ }^{4}$ En esta esquematización de ambos conceptos se omite que el habitus no es algo que sólo poseen los que pertenece a un campo determinado, sino algo que comparten todos aquellos que pertenecen a una cultura y forma parte del proceso de socialización que nos permite incorporarnos a un grupo a través de la asunción de los esquemas culturales que dicho grupo nos inculca. El habitus es un sistema de disposiciones duraderas y transferibles, "estructuras estructurantes", principios generadores y organizadores de las prácticas y las representaciones (Bourdieu 1991:92). El habitus, aunque nos es transferido, estructura nuestra experiencia de tal forma que no es perceptible, forma parte de nosotros en todo momento y sólo se puede poner en evidencia por un proceso reflexivo de extrañamiento.
} 
Para poder estudiar el campo, hay que considerar los principios metodológicos que el mismo Bourdieu planteó, especialmente la noción de la historización radical. Para Wacquant (2005), uno de los discípulos más notorios de Bourdieu, esta historización de las prácticas y el discurso que dan origen a cualquier hecho social, especialmente a los políticos, es un principio metodológico necesario tanto para el análisis de las trayectorias de los actores en el campo como de la constitución misma de éste, que evoluciona de acuerdo con las relaciones que establece con otros campos y actores que vulneran o modifican su autonomía relativa. En otras palabras, ningún hecho social, incluido el propio patrimonio, puede entenderse sin definir la evolución del espacio histórico que ocupa y ocupaba, y sin explicarlo en función de esta evolución. Esto implica también que para conocer un campo en la actualidad deberíamos conocer su evolución histórica.

La comprensión histórica de los hechos sociales, en especial del arte y otros fenómenos asociados al patrimonio cultural, está relacionada con su uso. Así, desde la teoría de campos, el patrimonio puede entenderse a través de su uso social. En el patrimonio podemos reconocer una función social específica que está relacionada con su poder simbólico, ya que éste surge para dar legitimidad a una visión de lo social y del orden sociopolítico, centrada en el valor de la identidad grupal y su existencia objetiva. Como Weber señaló (2004:150), sin importar los fundamentos objetivos de la creencia de una afinidad grupal, ésta tiene consecuencias en la formación política de una comunidad que busca mantener dicha creencia, esencial para su permanencia, independientemente de que la relación exista en realidad. Cualquier comunidad política busca inspirar el asenso sobre un origen común y lo alimenta con una memoria compartida que se materializa en un patrimonio común. Por ello, podemos suponer que el capital en juego en el campo del patrimonio será precisamente su capacidad de simbolizar y dar legitimidad a un orden sociopolítico.

\section{Patrimonio, restauración e interpretación}

Aquí haremos una breve consideración del papel de la restauración y la conservación en la disputa por el capital simbólico que contiene el patrimonio a partir de la reflexión de que, como disciplina, la Restauración no sólo se ocupa de la preservación física de los objetos, sino de fijar y hacer accesible una interpretación de su significado: entenderlo es una labor de interpretación que está sometida, como todas, a los cambios de cada momento histórico. Por ello, Berger (2000:23) señaló que la percepción del arte en la actualidad es completamente diferente de la de otros momentos históricos. La conservación de un objeto depende en buena medida de la posibilidad de identificar en él algún valor para una comunidad que haga deseable entonces su preservación. A éste se le asigna un peso objetivo para los interesados, el cual debe ser respetado o enriquecido por el restaurador, que asume como reales los sistemas de valoración que gravitan en torno del objeto $y$, para que sean consistentes con la interpretación que los interesados tienen sobre el objeto, adapta entonces las reglas de su propia práctica. Éstas, que incluyen los principios técnicos y éticos, también están conformadas por la cultura que los restauradores, como individuos y como gremio, incorporan en sus esquemas cognitivos para comprender el objeto del pasado.

Esta labor de interpretación y metainterpretación muchas veces se realiza de forma empírica, sin hacer consciente que la mirada del restaurador, la de los interesados y la de los creadores de un objeto están modeladas por el clima sociocultural donde se formaron y, por lo tanto, no son iguales. Al reconocer esto -lo que sería de gran ayuda al momento de decidir un proceso de restauración-, se asume que una intervención de restauración es una acción que incide en un recurso, el patrimonio, y que dicho capital está en disputa entre diferentes actores que pretenden su control.

Por lo anterior, se puede decir de manera provisional que la restauración implica una labor de interpretación de los objetos del pasado a los que una comunidad ha asignado valores que son consonantes con la cultura que ella misma incorpora y reconoce como suya, y que todo este esfuerzo está encaminado a controlar dichos objetos. Esa labor de interpretación no es menor, ya que es la que legitima la valoración que se puede hacer de un objeto o, para expresarlo en un sentido paradójico: para que el patrimonio lo sea, no basta con que una comunidad lo identifique como tal, sino que debe ser reinterpretado por los especialistas que se encargan, precisamente, de "poner en valor" los objetos que le dan sentido a aquélla. Este proceso sería entonces redundante, pero, en realidad, quien realiza la selección de los objetos que conforman el patrimonio es un grupo concreto dentro de cada sociedad, que será el que tenga mayor capacidad de interpretar e imponer sus interpretaciones sobre el valor de los objetos. Así, en toda cultura existe un grupo que define los significados y valores que el resto de la sociedad asume como normas de sentido. La definición que hace este grupo no necesariamente va a partir de un consenso, sino más bien, de una disputa por el reconocimiento necesario para convertirse en un productor de sentido o productor cultural. Lo podemos ver, por ejemplo, en el campo mismo del arte o en el académico de la cultura, donde los actores debaten entre sí la legitimidad de sus representaciones e interpretaciones, pero siempre según las reglas que ellos mismos crean y aceptan.

Esto último pone en evidencia una situación que el propio Bourdieu ya había señalado (1993:115): la oposición entre un campo restringido de producción cultural, donde los productores son sus consumidores y el campo de producción a gran escala, destinado al público en general, dominado por los dueños de los medios de pro- 
ducción y difusión de los bienes culturales que emplean o no a los productores culturales del campo restringido. En el caso de los bienes culturales, éstos se constituyen como patrimonio a través de un fenómeno de producción simbólica que los transforma de objetos a codificadores de una cultura. En general, este proceso, así como la distribución y la reproducción de las interpretaciones que genera, ha estado dominado por especialistas (arqueólogos, historiadores, museólogos, restauradores, conservadores, gestores culturales, etc.) que disputan para ellos mismos el derecho de interpretar los objetos que a la postre comparten con el público general.

Después, las interpretaciones de los especialistas pasan al resto de la sociedad a través de las instituciones culturales del Estado o de los Ilamados mass media, o medios masivos de comunicación. En ambos casos, el acceso al patrimonio se usa como medio para la divulgación de un discurso que interpretan los especialistas y se asimila a los objetos a través de las pautas de lectura, presentación y acceso que ellos generan para el resto de los actores. Generalmente, este discurso incluye elementos sobre la afirmación de un pasado común y un orgullo inherente a dicho pasado. Esto tiene la finalidad de educar al ciudadano, infundiendo en él valores que avalan la organización de su sociedad o régimen político y finalmente justifican y legitiman la dominación de aquellos que controlan el campo de la producción cultural. Aunque en teoría el patrimonio cultural es una propiedad colectiva de los ciudadanos, el acceso estará generalmente mediado por aparatos culturales diseñados para su presentación, interpretación y consumo, entre los que destaca el museo.

De esta manera, tendríamos que destacar como propiedades del campo de la reproducción y circulación de bienes culturales del pasado: i) la producción o reproducción de interpretaciones de éstos, que es una prerrogativa de los especialistas que ejercen en un campo restringido del que salen las interpretaciones al campo general de producción cultural para su consumo por parte del público; ii) la distribución en el campo general cultural, la cual está mediada por instituciones que determinan el uso que se dará a las interpretaciones producidas por los especialistas y el público al que se destinarán como parte de un política cultural. En consecuencia, el consumo de los bienes culturales del pasado estará entonces doblemente condicionado: por un lado, por la posibilidad de acceder a ellos a través del mercado de bienes culturales controlado por las instituciones, $y$, por el otro, por la necesidad de conocer los códigos de interpretación generados por los especialistas. ¿Es posible entonces que los usuarios, custodios o consumidores del patrimonio puedan recibir algún beneficio de éste sin depender de la mediación que hacen para ellos los especialistas y las instituciones culturales? ¿Hay forma de que puedan superar, o al menos contrarrestar, la doble condicionante que se les impone y acceder a los bienes culturales con interpretaciones propias?

\section{El restaurador intérprete ${ }^{5}$}

Varios autores han reconocido en la restauración un proceso de interpretación del patrimonio que, en principio, busca reconocer en los objetos su valores "inherentes", provocando una reificación de los mismos. Para la ortodoxia de la disciplina, la restauración condiciona el tratamiento de un objeto de acuerdo con el objeto mismo (Jokilehto 2002). Sin embargo, también se ha admitido ya que la acción de restauración tienen la capacidad de modificar el objeto y, con ello, su significado (Rocha Reyes y Vega Cárdenas 1997). Aun las intervenciones menos "invasivas", o una aparentemente inocua "conservación preventiva", provocan cambios en el valor simbólico del objeto por el simple hecho de plantear su conservación. Ésta implica una revalorización a ojos del público y de los especialistas que altera el significado del objeto. A pesar del imperativo por la autenticidad que impregna a toda la teoría de la restauración, la conservación del patrimonio no pretende mantener el significado del objeto, sino mejorarlo. Este proceso de mejora depende del uso adecuado de las reglas del juego comunicativo establecido, por lo que terminan por reproducir los esquemas cognitivos hegemónicos, especialmente entre aquellos que menos herramientas o recursos tienen para participar en la interpretación de los objetos o para proponer intervenciones alternativas.

La valoración que provoca la conservación agrega significados al objeto, reafirmando el valor del patrimonio para el presente mediante el establecimiento de relaciones con el orden social imperante, del que recibe legitimación, a la vez que es legitimado. Por ello podemos decir que la protección del patrimonio es una labor conservadora en el sentido político, ya que termina por legitimar un orden social dado, más que cuestionarlo o, al menos, analizarlo.

Vega Cárdenas (2008) ya ha planteado el grave problema que significa el hecho de que la restauración se dedique casi siempre a reproducir un discurso hegemónico, que además le es impuesto por otros actores. Al mismo tiempo señala que es un imperativo moral plantear cómo la restauración puede contribuir a una "revolución simbólica", difícil de imaginar, cuando conservamos bajo la consigna de que el significado del patrimonio sólo puede ser apreciado por medio de la educación de los usuarios en el mismo sistema de significados que se pretende revolucionar. En otras palabras, pretendemos que los ciudadanos interpreten el patrimonio de formas novedosas

\footnotetext{
${ }^{5}$ Para este apartado tomamos el título de un interesante debate que se dio en el número 1 de Intervención y que abunda sobre el papel y las capacidades del restaurador como intérprete. Basta señalar en nuestro caso que apoyamos la tesis de que el restaurador realiza interpretaciones, pero que en este momento nos abstenemos de emitir un juicio sobre el valor cognitivo o estético de éstas para centramos en sus implicaciones socioculturales.
} 
aprendiendo a interpretarlo como nosotros lo interpretamos. Éste es el presupuesto subyacente en las iniciativas de educación patrimonial y en el asistencialismo intelectual, incluido en muchos discursos museográficos e iniciativas de conservación "comunitaria". Berger (2000:7) señalaba que "el arte del pasado está siendo mistificado porque una minoría privilegiada se esfuerza por inventar una historia que justifique retrospectivamente el papel de las clases dirigentes, cuando tal justificación no tiene ya sentido en términos modernos". Más que una revolución simbólica, la ortodoxia de la conservación del patrimonio plantea una inculturación de los que, desde la perspectiva del especialista, no son capaces de entender su patrimonio. Esta situación subsiste; en México, especialmente a raíz de la estructura de las instituciones culturales del país, así como de las políticas culturales, que han privilegiado la participación del Estado en la construcción e interpretación del patrimonio. En el caso particular del campo de la arqueología, Vázquez (2003) ha hecho un completo análisis de la forma en que la disciplina en México se convirtió en buena medida en un generador de propaganda para el régimen.

Opuesta a esta historia de determinaciones, podemos observar muchas veces que, a pesar de que el consumidor de bienes culturales patrimoniales se apega a ciertas pautas de comportamiento inculcadas por los mecanismos disciplinantes más diversos, desde la escuela, hasta los medios masivos, también presenta comportamientos "anómalos". Desde las resignificaciones de figuras aparentemente sacralizadas, como la Virgen de Guadalupe, o el surgimiento de nuevos patrones de significado, como la rica iconografía de los cultos populares (la devoción a la Santa Muerte o Martín Valverde, por ejemplo), vemos con frecuencia que los consumidores o usuarios del patrimonio revierten las interpretaciones convencionales o crean las suyas, que florecen, aunque a los especialistas puedan resultar chocantes, de forma aparentemente impredecible. Hace poco, durante una visita hecha por alumnos de la Licenciatura de Restauración de la Universidad Autónoma de San Luis Potosí a una ex hacienda casi en ruinas, algunos jóvenes de la comunidad que vive en torno del edificio mencionaron que sería interesante usarlo como centro de desintoxicación, ya que ahí era donde se juntaban a consumir narcóticos. La paradoja es muy estimulante, ya que si bien tratan de crear un espacio autodisciplinante, es notorio que, al menos en el plano simbólico, no les interesan las interpretaciones canónicas de un espacio de ese tipo sino su utilidad a escala local.

¿Cómo se dan estas subversiones del orden simbólico que los restauradores, arqueólogos e historiadores imponen, por ejemplo, a una zona arqueológica y que después, alegremente, los guías de la misma la convierten en la base de operaciones de los extraterrestres? ¿Dónde debería estar nuestra fidelidad: con la construcción de objetividad sancionada por el campo científico o con las infinitas variaciones y desviaciones que los usuarios pue- den crear? El restaurador más ortodoxo diría que está en el objeto; pero, al final, éste sólo tiene sentido cuando lo interpretamos y nuestra interpretación tendrá un efecto en cualquiera de estos dos sentidos. Desde la perspectiva del campo, el problema radica en que la restauración como práctica no hace más que poner en evidencia un habitus, producto de la interiorización de las estructuras que hacen que el patrimonio se adecue a las estructuras sociales ya existentes y hegemónicas. El que no puede entender las prácticas relacionadas con el patrimonio debe entonces aprender a entenderlas e interiorizarlas, y queda entonces subsumido en la misma estructura que las produjo. No hay posibilidad de disenso; si queremos entender el patrimonio de otra forma, tendríamos que subvertir todo el sistema que le da coherencia y sentido, para entonces asumir que conservamos objetos que sólo tienen valor porque nos han enseñado que lo tienen. ¿Hay alguna forma de salvar esta situación, de permitir que el patrimonio siga teniendo una función y, al mismo tiempo, que no sea solamente un dispositivo de poder con el que un grupo impone su visión de pasado?

En los últimos años, especialmente a partir de la década de 1980, hemos podido verificar una paulatina reducción del papel del Estado en todos los ámbitos, especialmente en el cultural. Incluso organismos como el Banco Mundial emiten en esta época recomendaciones para adelgazar al Estado y hacer a los ciudadanos copartícipes de los procesos de gobierno. El proceso, por supuesto, afectó al campo de la cultura en México, generando diferentes corrientes con la vocación de que los ciudadanos participen en la construcción, la interpretación y el uso del patrimonio. Estos procesos participativos, muchas veces contestatarios a los poderes formales y en representación de grupos subalternos o marginados, han iniciado la revalorización de patrimonios que se encontraban muchas veces fuera del circuito del patrimonio consagrado por el Estado (véase Pérez Ruiz 2008). Sin embargo, la participación no es necesariamente sinónimo de rompimiento con las estructuras de interpretación y sentido hegemónicas, aunque sí ofrece una referencia para la redefinición de las políticas culturales (García Canclini 1997). Las políticas participativas en otras esferas ya han sido abordadas a mayor profundidad, revelando que si bien contribuyen a la construcción de marcos democráticos, son inútiles sin el reconocimiento e institucionalización por parte de la sociedad. Por otro lado, en muchos casos propician la formación de grupos restringidos que cooptan la participación, ya que no llevan a la esfera pública al ciudadano corriente, sino a los que ya tomaban parte en cierta medida en la toma de decisiones y cuya participación consideran valiosa (Abers 2003:337; Canto Chac 2007). En el campo de la cultura, al menos en el caso de la Huatápera de Uruapan, se han observado situaciones en las que la participación en los procesos de gestión del patrimonio llega a generar divisiones entre diferentes sectores de la población o el apoyo a movimientos de reivindicación 
étnica que alimentan una interpretación excluyente del patrimonio (Jiménez Ramírez 2010).

Al margen de los esfuerzos institucionalizados, muchos grupos interpretan el patrimonio de formas más o menos espontáneas, generando alternativas que incluso pueden chocar con la noción más aceptada del patrimonio. La pregunta es si éstas son una opción para conservar el patrimonio y, al mismo tiempo alterar su efecto de dominación, y si pueden o no considerarse dentro del horizonte de acción de la restauración. Para ello, primero tendríamos que entender la naturaleza de estas prácticas y si son realmente alternativas a las estructuras que se imponen en las interpretaciones canónicas, o sólo variaciones chapuceras que dan nuevos colores pero que cambian muy poco la función hegemónica del patrimonio.

Desde la sociología tendríamos que preguntarnos si existe esta posibilidad, considerando que para el propio Bourdieu, si bien existe el cambio y la movilidad social, ésta difícilmente puede atribuirse a las prácticas o a la agencia de los sujetos, sino más bien, a las coyunturas de cambio en las estructuras (Certeau 1996:68). Michel de Certeau (1996), en su crítica a la visión de Bourdieu, propone que no obstante que existen estrategias generales de dominación, también los sujetos son capaces de generar su propias tácticas para confrontarlas. Un ejemplo de esto es la forma en que los individuos discurren su vida en las ciudades, a pesar de que éstas son el lugar donde se despliegan los dispositivos de poder en el espacio, condicionando la forma en que las habitamos (Foucault 2006:36). Cuando, pese a que se asume una organización dada del espacio, se camina por la ciudad, en ocasiones el caminante la actualiza y subvierte; con sus propios y particulares modos de caminar, opone una resistencia al poder disciplinante, por más que discurra a través de él. En el caso del patrimonio, esas tácticas no tienen que ser una invención absoluta como no lo es caminar por la ciudad; tal vez sólo pasen por un extrañamiento debido a la situación del objeto del pasado. Si el observador se cuestiona no qué significa para él un objeto del pasado, sino cómo es que está ahí, entonces se podría pensar en un rompimiento en las formas en las que éste lo incorpora a su horizonte cultural. El problema radica en cómo transformar el patrimonio, de un recurso para la confirmación de la identidad, en un dispositivo que rompa la homogeneidad de la memoria y provoque sobresaltos al observar el presente.

La cuestión, en un primer instante, no suena sencilla, ya que se opone a uno de los principios que se asumen de la misma práctica de conservar: conservamos algo porque nos hace referencia a nosotros mismos y no porque nos haga cuestionarnos qué ha sido del pasado.

Muñoz Viñas (2003) menciona que los objetos en sí tienen importancia por los significados y usos que las personas asignan a éstos y por los valores que representan. Habría que agregar que ésta también radica en la forma en que pueden ser usados, en el sentido amplio del concepto, como recurso. En ese sentido, y aunque la labor del restaurador siempre será interesada ya que defiende sus propias conveniencias disciplinares y personales, puede pensarse en restauraciones más neutras que permitan a las personas hacer uso del patrimonio según sus intereses y no sólo de acuerdo con los intereses hegemónicos o los de un grupo que domina la participación. Esto hace cuestionar el principio de seguridad que da valor al patrimonio como uno de los elementos centrales en la pretensión de hacer del pasado un "lugar seguro". El patrimonio ayuda a hacer del pasado un sitio habitable donde podemos saber con certeza de dónde venimos y por qué las cosas son como son.

Si queremos cambiar el uso del patrimonio, tendríamos que propiciar entonces la generación de una incertidumbre interpretativa entre aquellos que se enfrenten a él o al menos, permitirles que asuman una interpretación propia. En lugar de pensar el patrimonio como un elemento de identificación y pretender que la gente también se identifique con él, podríamos empezar por cuestionar qué hace la gente con él. En este terreno tal vez los museólogos tienen algo de camino adelantado, pero también es cierto que se han preocupado por saber qué hace la gente en los museos, pero muy poco por entender la relación de los usuarios con la ciudad patrimonial, las zonas arqueológicas o los novedosos paisajes culturales. Para nuestra sorpresa, el patrimonio convive todo el tiempo con personas que no lo ven como un repositorio de sentido histórico: mucha gente vive en él y de él. Esta situación tensa el precario equilibrio entre el control gubernamental de patrimonio y el uso libre de éste, para abrir otros debates sobre la conveniencia de permitir su aprovechamiento como recurso económico y los efectos que esto puede tener para su conservación. La explotación de los atractivos culturales ahora se enfrenta con la necesidad de preservar la "autenticidad" del atractivo, al mismo tiempo que debe conciliar a un creciente número de visitantes que alteran por completo la forma de percibir un objeto o sitio patrimonial. Estas tensiones hacen que en muchos lugares los bienes patrimoniales se conviertan en objeto de disputas, por lo que su control puede convertirse en un objetivo político que va más allá de la necesidad de dotar de identidad a los ciudadanos.

\section{Conclusiones}

A pesar de proyectos, cada vez más frecuentes, en los que el patrimonio se pone a discusión con sus usuarios en todo el mundo, aún está por verse si este proceso terminará con las tensiones que surgen entre los actores que disputan su control, que son las que finalmente le dan significado, y no las propiedades inmanentes que éstos le puedan asignar a los objetos. Por ello es posible imaginar otros sentidos en el patrimonio, ya que su interpretación es, al final, el resultado de las relaciones entre los actores que participan de su definición y de los intereses que éstos 
defienden. Las filas de estudiantes copiando las cédulas de una exposición monumental que dicen lo mismo que les han repetido en la escuela son la manifestación de una práctica que busca en el patrimonio una certeza de que las cosas deben ser así. La confrontación con los especialistas o los desacuerdos entre ellos revelan movimientos dentro del campo del patrimonio que están alterando su sentido. Si nuestro interés es contribuir a cambiar la forma en que se ve el patrimonio y, en general, en la que lo construye la sociedad, tal vez deberíamos dejar de creer que los usuarios deben "copiar" nuestras interpretaciones u observar los objetos desde una perspectiva atemporal para que puedan "leerlos" con facilidad. Tal vez es momento de dejar que el usuario se pregunte por qué las cosas están en ese estado y si él puede hacer algo para mejorarlo, antes de decirle qué es lo que tiene que hacer.

\section{Referencias}

\section{Abers, Rebecca Neaera}

2003 "Reflexiones sobre los factores que dan lugar al gobierno participativo con poder de decisión", en Erik Olin Wright y Archon Fung (eds.), Democracia en profundidad. Nuevas formas institucionales de gobierno participativo con poder de decisión, I, Bogotá, Universidad Nacional de Colombia (Utopías Reales, 1): 337-349.

Ariel Olmos, Héctor 2004 Cultura: El sentido del desarrollo, México, ConacultaInstituto Mexiquense de Cultura (Intersecciones, 2).

Arrieta Urtizberea, Iñaki (ed.) 2008 Participación ciudadana, patrimonio cultural y museos: Entre la teoría y la praxis, Bilbao, Universidad del País Vasco, documento electrónico disponible en [http://www.argitalpenak.ehu.es/p291-content/es/contenidos/libro/se_indice_arte/ es_arte/adjuntos/particip.pdf], consultado el 15 de abril de 2009.

Berger, John 2000 Modos de ver, Barcelona, Gustavo Gili.

Bourdieu, Pierre

1991 El sentido práctico, Madrid, Taurus (Humanidades). 1993 "The market of symbolic goods", en The Field of Cultural Production: Essays on Art and Literature, Randal Johnson (ed.), Nueva York, Columbia University Press: 112-141. 1998 La distinción. Criterios y bases sociales del gusto, Madrid, Taurus (Ensayistas, 259).

Brandi, Cesare 1996 Teoría de la restauración, Madrid, Alianza.

Canto Chac, Manuel

2007 "Participación ciudadana: La gobernanza de las sociedades complejas", en Democracia y gobernabilidad, José Luis Calva (ed.), México, unam/ Miguel Ángel Porrúa/ H. Cámara de Diputados, LX Legislatura (Agenda para el desarrollo, 15): 140-159.

Certeau, Michel de

1996 La invención de lo cotidiano, vol. I, Guadalajara, ITESo/ UIA/ Centro de Estudios Mexicanos y Centroamericanos.
Conferencia de Nara sobre la Autenticidad

1994 Documento de Nara sobre la autenticidad, Raymond Lemaire y Herb Stovel (eds.), UNESCO, s. I.

Fontal Merillas, Olaia

2003 La educación patrimonial, Teoría y práctica en el aula, el museo e internet, Gijón, Trea (Biblioteconomía y Administración Cultural, 87).

Foucault, Michel

2006 "Clase del 11 de enero de 1978" en Seguridad, territorio y población. Curso en el Collège de France, 1977-1978, Buenos Aires, FCE (Sección de Obras de Sociología): 15-45.

García Canclini, Néstor

2006 [1979] La producción simbólica. Teoría y método en sociología del arte, 7a. ed., Buenos Aires, Siglo XXI Editores. 1997 "El patrimonio cultural de México y la construcción imaginaria de lo nacional", en El patrimonio nacional de México, vol. I, México, Conaculta/FCE (Biblioteca Mexicana 1): 57-86.

ICOMOs Australia

1999 Carta del ıCOM Os Australia para sitios de significación cultural.

Jiménez Ramírez, Mauricio Benjamín

2010 "La construcción del discurso del patrimonio en la Huatápera de Uruapan", tesis de maestría en Comunicación de la Ciencia y la Cultura, Guadalajara, ITESO.

Jokilehto, Jukka 2002 A History of Architectural Conservation, Oxford, Butterworth-Heinemann.

Muñoz Viñas, Salvador

2003 Teoría contemporánea de la Restauración, Madrid, Síntesis.

Pérez Ruiz, Maya Lorena

2008 "La museología participativa: ¿Tercera vertiente de la museología mexicana?", en Cuicuilco vol. 15, núm. 44: 87110.

Reunión sobre Conservación y Utilización de Monumentos y Lugares de Interés Histórico y Artístico

1967 Normas de Quito.

Rocha Reyes, Juan Manuel y Alfredo Vega Cárdenas

1997 "Iconografía y Restauración: El estudio iconográfico en la restauración de los bienes culturales sacros", licenciatura en Restauración de Bienes Muebles, México, ENCRYM-INAH.

Tagliorette, Alicia Renée, Patricia Sampaoli, Ana María Ibarroule y Elio Reynoso

2008 "El difícil equilibrio entre el fortalecimiento institucional y la gobernabilidad de un proceso de gestión sustentable del patrimonio cultural, en una experiencia desarrollada en el noreste de la provincia de Santa Cruz en la Patagonia argentina", en III Congreso Patrimonio Cultural y Cooperación al Desarrollo, Gaspar Muñoz Cosme y Cristina Vidal Lorenzo (eds.), México, Instituto Universitario de Restauración del Patrimonio/ UPV, marzo: 301-309.

Teixeira, Simonne

2006 "Educación patrimonial: Alfabetización cultural para la ciudadanía", en Estudios pedagógicos, Valdivia, 32: 133-145. 
Tenopala García, Óscar

1998 "Metodología participativa en el proceso de restauración del patrimonio cultural", El Correo del Restaurador, núm. 7, documento disponible en [http://www.conservacionyrestauracion.inah.gob.mx/html/correo_restaurador/html/ CORE07.html].

Vega Cárdenas, Alfredo

2008 "El oficio de restaurador como instrumento de destino: elementos teóricos y metodológicos para una sociología de la restauración", tesis de maestría en Filosofía Social, Guadalajara, ITESO.

\section{Resumen}

A partir del análisis de la valoración, o puesta en valor, del patrimonio que se realiza antes de la conservación de un objeto, se plantea observar este proceso como parte de otros fenómenos socioculturales más amplios que se pueden modelar con ayuda del concepto de campo de poder de Bourdieu. El patrimonio, es entonces el producto cultural de la interpretación del pasado y la cultura, realizada por los especialistas, difundida por las instituciones y asimilada por la población como parte de la construcción de la legitimidad de una cultura, muchas veces hegemónica. Se mencionan algunas alternativas a este modelo y la necesidad de repensar el patrimonio desde la capacidad de los sujetos de reinterpretarlo y no sólo desde los grupos, a pesar de que esto vaya contra el mismo concepto de patrimonio como uno de los fundamentos de la construcción de la identidad grupal

\section{Palabras clave}

Campo de poder, valoración, dominación, patrimonio, producción cultural
Vázquez León, Luis

2003 El leviatán arqueológico: antropología de una tradición científica en México, 2a. ed., México, CIESAS/ Miguel Ángel Porrúa.

Wacquant, Loïc

2005 "Indicaciones sobre Pierre Bourdieu y la política democrática", en Loïc Wacquant, El misterio del ministerio. Pierre Bourdieu y la política democrática, Barcelona, Gedisa (Sociología): 23-42.

Weber, Max

2004 The Essential Weber: A Reader, Londres, Routledge.

\section{Abstract}

The assessment of heritage, made before any conservation process, is the subject of this paper, which proposes how this assessment becomes part of a major sociocultural phenomenon that can be modeled with Bourdieu's field of power theory. Heritage is defined as a cultural produce that is obtained from the interpretation of past and culture, which is made by experts, transmitted by institutions and assimilated by people as part of a legitimizing discourse about a culture that is often hegemonic. We analyze some alternatives to this model and emphasize the need to rethink heritage from the capacity of people to make new interpretations, and not only as a function of hegemonic groups. This goes against one of the fundamentals of the heritage concept: its role as an identity constructor.

\section{Keyword}

Field of power, Assessment, Domination, Heritage, Cultural product. 